Virginia Commonwealth University

VCU Scholars Compass

Surgery Publications

Dept. of Surgery

2017

\title{
A pilot study to measure dynamic elasticity of the bladder during urodynamics
}

Andrew F. Colhoun

Virginia Commonwealth University

Adam P. Klausner

Virginia Commonwealth University, adam.klausner@vcuhealth.org

Anna S. Nagle

Virginia Commonwealth University, asnagle@vcu.edu

See next page for additional authors

Follow this and additional works at: http://scholarscompass.vcu.edu/surgery_pubs

Part of the Surgery Commons

(C) 2016 Wiley Periodicals, Inc.

\section{Downloaded from}

http://scholarscompass.vcu.edu/surgery_pubs/27

This Article is brought to you for free and open access by the Dept. of Surgery at VCU Scholars Compass. It has been accepted for inclusion in Surgery Publications by an authorized administrator of VCU Scholars Compass. For more information, please contact libcompass@vcu.edu. 
Authors

Andrew F. Colhoun, Adam P. Klausner, Anna S. Nagle, Ashley W. Carroll, Robert W. Barbee, Paul H. Ratz, and John E. Speich 


\title{
A Pilot Study to Measure Dynamic Elasticity of the Bladder During Urodynamics
}

\author{
Andrew F. Colhoun, ${ }^{1}$ Adam P. Klausner, ${ }^{1}$ Anna S. Nagle, ${ }^{2}$ Ashley W. Carroll, ${ }^{3}$ Robert W. Barbee, ${ }^{4}$ \\ Paul H. Ratz, ${ }^{5}$ and John E. Speich ${ }^{2 *}$ \\ ${ }^{1}$ Division of Urology/Department of Surgery, Virginia Commonwealth University School of Medicine, Richmond, Virginia \\ ${ }^{2}$ Department of Mechanical and Nuclear Engineering, Virginia Commonwealth University School of Engineering, \\ Richmond, Virginia \\ ${ }^{3}$ Department of Obstetrics and Gynecology, Virginia Commonwealth University School of Medicine, Richmond, Virginia \\ ${ }^{4}$ Departments of Emergency Medicine and Physiology and Biophysics, Virginia Commonwealth University School of Medicine, \\ Richmond, Virginia \\ ${ }^{5}$ Departments of Biochemistry and Molecular Biology and Pediatrics, Virginia Commonwealth University School of Medicine, \\ Richmond, Virginia
}

\begin{abstract}
AIMS: Previous studies using isolated strips of human detrusor muscle identified adjustable preload tension, a novel mechanism that acutely regulates detrusor wall tension. The purpose of this investigation was to develop a method to identify a correlate measure of adjustable preload tension during urodynamics. METHODS: Patients reporting urgency most or all of the time based on ICIq-OAB survey scores were prospectively enrolled in an extended repeat fill-and-empty urodynamics study designed to identify a correlate of adjustable preload tension which we now call "dynamic elasticity." Cystometric capacity was determined during initial fill. Repeat fills to defined percentages of capacity with passive emptying (via syringe aspiration) were performed to strain soften the bladder. A complete fill with active voiding was included to determine whether human bladder exhibits reversible strain softening. RESULTS: Five patients completed the extended urodynamics study. Intravesical pressure $\left(\mathrm{p}_{\mathrm{ves}}\right)$ decreased with subsequent fills and was significantly lower during Fill 3 compared to Fill $1(P=0.008)$, demonstrating strain softening. Active voiding after Fill 3 caused strain softening reversal, with $\mathrm{p}_{\mathrm{ves}}$ in Fill 4 returning to the baseline measured during Fill $1(P=0.29)$. Dynamic elasticity, the urodynamic correlate of adjustable preload tension, was calculated as the amount of strain softening (or its reversal) per \% capacity ( $\Delta$ average $\mathrm{p}_{\mathrm{ves}}$ between fills/ $\Delta \%$ capacity). Dynamic elasticity was lost via repeat passive filling and emptying (strain softening) and regained after active voiding regulated the process (strain softening reversal). CONCLUSIONS: Improved understanding of dynamic elasticity in the human bladder could lead to both improved sub-typing and novel treatments of overactive bladder. Neurourol. Urodynam. 36:1086-1090, 2017. (c) 2016 Wiley Periodicals, Inc.
\end{abstract}

Key words: biomechanics; compliance; overactive detrusor function; urinary bladder; urodyanmics

\section{INTRODUCTION}

Acute changes in detrusor wall tension are important in overactive bladder syndrome (OAB) in that increased wall tension per unit volume may lead to higher-than-normal sensations of urgency. Wall tension is affected by the filling pressure, bladder geometry (volume and shape), and the material properties of the bladder wall. During standard clinical urodynamics (UD), the bladder material property calculated during the filling phase is a single, "static," compliance value, defined as the ratio of the change in volume to the change in pressure over the entire fill. ${ }^{1}$ Changes in bladder compliance are only expected through chronic disease processes causing increased collagen deposition and decreased elasticity. ${ }^{2,3}$

We now introduce the term "dynamic elasticity" which refers to a material property of detrusor smooth muscle (DSM) responsible for acute changes in wall tension that occur from one fill to another during repeat bladder filling and passive emptying. Dynamic elasticity occurs due to strain softening. A common example of strain softening is observed when a latex balloon is repeatedly stretched and released prior to inflation. The result is a reduction in stiffness, which produces lower wall tension during filling and makes the balloon easier to inflate. The strain softening that occurs in a latex balloon is irreversible, so that once the balloon is stretched it can never regain its original wall tension for a given volume and shape. This behavior is clearly differentiated from the reversible and actively regulated strain softening that occurs in detrusor smooth muscle (DSM) from both humans and other mammalian species. ${ }^{4-11}$

In these earlier in vitro studies, an acute reduction in DSM tension (increased compliance) was identified after strain softening, which was reversed with active muscle contraction at shorter muscle lengths. ${ }^{4-11}$ Strain softening is quantified as adjustable preload tension in DSM strips, and strain softening reversal is regulated by rho-kinase in DSM from multiple species, including humans. ${ }^{4,7,12}$ We hypothesize that preclinical strain softening measured as adjustable preload tension in

Dr. Lori Birder led the peer-review process as the Associate Editor responsible for the paper.

Potential conflicts of interest: Drs. Colhoun, Barbee, and Ratz have nothing to disclose. Drs. Klausner, Nagle, Carroll, and Speich report grants from National Institutes of Health (NIH) during the conduct of the study.

Grant sponsor: Virginia Commonwealth University Presidential Research Quest; Grant sponsor: NIH; Grant number: R01DK101719

* Correspondence to: John E. Speich, Ph.D., Department of Mechanical and Nuclear Engineering, Virginia Commonwealth University, 401 West Main Street, PO Box 843015, Richmond, VA 23284. E-mail: jespeich@vcu.edu

Received 13 April 2016; Accepted 10 May 2016

Published online 31 May 2016 in Wiley Online Library

(wileyonlinelibrary.com)

DOI 10.1002/nau.23043 
DSM strips can be identified clinically as "dynamic elasticity" during UD testing.

The present pilot study utilizes an innovative repeat passive filling and emptying protocol to test whether dynamic elasticity can be identified during clinical UD testing. Because strain softening is a regulatable biomechanical process affecting tension in DSM, identification and characterization of dynamic elasticity during UD may lead to improved understanding of the pathophysiology of both $\mathrm{OAB}$ and detrusor underactivity, and ultimately to the development of novel treatments for both of these conditions.

\section{MATERIALS AND METHODS}

This study was approved by the Institutional Review Board of Virginia Commonwealth University. Individuals with OAB, defined as International Consultation on Incontinence Ouestionnaire (ICIq-OAB question $5 \mathrm{a} \geq 3$, urgency most or all of the time) were prospectively enrolled. Pertinent demographic data, a complete ICIq-OAB survey, and a patient-reported 3-day void diary were obtained from each patient prior to UD testing.

\section{Urodynamics Testing}

A UD protocol was designed to directly translate our preclinical methodology established to quantify adjustable preload tension in isolated DSM strips ${ }^{4-11}$ to human UD studies. To accomplish this, an extended repeat fill protocol with passive emptying and active voiding for specific fills was developed (Fig. 1A). An initial UD study was performed per best practice guidelines for clinical purposes and to determine maximum cystometric capacity (CCap). ${ }^{13}$ For the initial fill, the fill rate was set at $10 \%$ of patient-reported maximum voided volume per minute on a 3-day void diary completed prior to the study. When the patient acknowledged that they had reached their maximum capacity (defined as inability to tolerate further filling or the presence of an involuntary bladder contraction), filling was stopped and the patient voided. Any post void residual was removed through the catheter via syringe aspiration, and the bladder was confirmed to be empty by an ultrasound technologist with transverse and sagittal midline suprapubic images taken at the onset and conclusion of each fill-and-empty cycle with a Philips Epiq 7 system with a 1-5 MHz abdominal probe (Amsterdam, The Netherlands). For all subsequent fills, CCap was defined as the sum of the voided volume and post void residual from the initial fill. Multichannel digital pressure and flow data acquisition was performed at $10 \mathrm{~Hz}$ via an Aquarius $\mathrm{TT}^{\mathrm{TM}}$ system (Laborie, Toronto). Four repeat fills were then initiated at a rate $10 \%$ CCap $/ \mathrm{min}$ as follows: (i) fill to $30 \%$ CCap and passively empty; (ii) fill to $60 \%$ CCap and passively empty; (iii) fill to CCap and void (voluntary or involuntary); and (iv) fill to 60\% CCap and void (Fig. 1A). Fills 1 and 2 were set to $30 \%$ and $60 \%$ CCap, respectively, to avoid triggering an active contraction which would limit the ability to identify the process of strain softening. Also, previous studies have used 33\% stretches to effectively strain softening DSM strips. $^{9}$

Passive emptying at the end of Fills 1 and 2 was performed via syringe aspiration through a three-way stopcock in series with the infusion catheter so as not to alter calibrated pressures. The bladder was confirmed to be empty by ultrasound as previously described. Repeat Fills 1-2 were performed to progressively strain soften the bladder with incrementally increasing volumes (Fig. 1A). In a fluid-filled thinwalled vessel, pressure is directly related to wall tension by the Law of Laplace (tension $\propto$ pressure $\times$ radius); therefore, strain
A

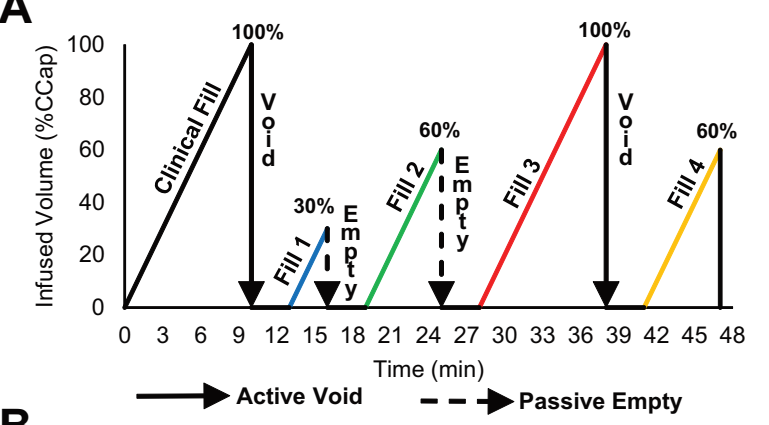

B

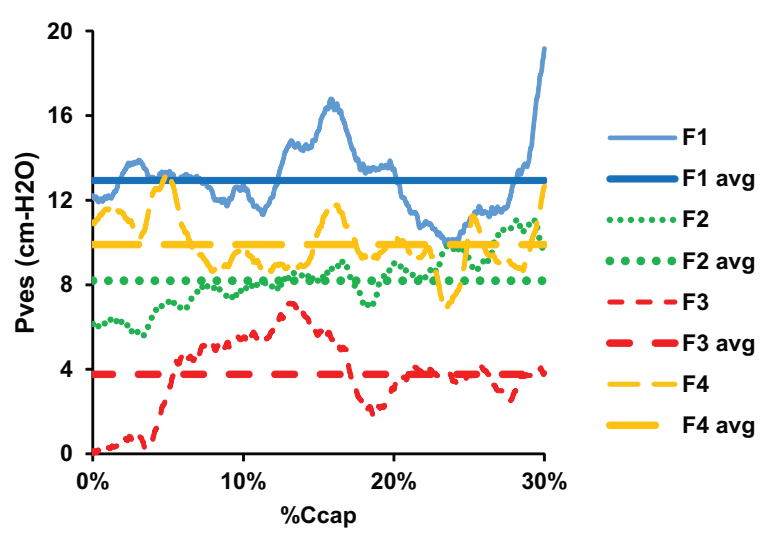

Fig. 1. A Repeat-fill urodynamics protocol. Initial clinical fill used to establish cystometric capacity (CCap). Bladder was filled with incrementally increasing volumes to strain soften the detrusor. Passive emptying via syringe aspiration was performed after Fills 1 and 2. Patient voided after Fill 3 to abolish dynamic compliance changes induced by strain softening B Superimposed $p_{v e s}$ tracings for Fills 1-4 (F1-F4) of the repeat-fill urodynamics protocol from an example participant. The $p_{\text {ves }}$ values for all fills were shifted by a single constant to set the minimum $p_{\text {ves }}$ for the each participant to zero. Tracings are overlaid with average $\mathrm{p}_{\mathrm{ves}}$ between $0 \%$ and $30 \%$ CCap for each fill.

softening reflected by decreased wall tension should be identified by comparing the change in luminal pressure between fills. For the present protocol, the degree of strain softening was determined by comparing average intravesical pressure $\left(p_{v e s}\right)$ from $0 \%$ to $30 \%$ CCap in subsequent passive fills. The expected result was a progressive decrease in pressure $\left(p_{v e s} 1>p_{v e s} 2>p_{v e s} 3\right)$ consistent with strain softening (Fig. 1B). The active void after Fill 3 was expected to reestablish the lost tension and demonstrate the reversibility of strain softening because preclinical studies show that active contraction reverses strain softening in DSM strips. ${ }^{4-11}$ Thus, Fill 4 was expected to demonstrate $p_{\text {ves }}$ similar to that observed in Fill 1.

\section{Justification for Using $\mathrm{p}_{\mathrm{ves}}$ Instead of $\mathrm{p}_{\mathrm{det}}$}

Detrusor pressure $\left(p_{\text {det }}\right)$ is determined by subtracting abdominal pressure $\left(p_{a b d}\right)$ from $p_{v e s}$. The role of the $p_{d e t}$ calculation is to remove confounding pressure transients from the abdominal compartment (i.e., cough, respirations, and rectal contractions) thereby preventing misinterpretation. Such events can appear as elevations in both $p_{v e s}$ and $p_{a b d}$ (Fig. 2, points $A$ and $B$ ) but are eliminated on $p_{\text {det }}$ due to subtraction (Fig. 2, point C). However, if $p_{a b d}$ elevates with a magnitude significantly greater than that seen in $p_{\text {ves }}$ (Fig. 2, points $D$ and $\mathrm{E}$ ), then the resulting $\mathrm{p}_{\text {det }}$ returns negative (Fig. 2, point $\mathrm{F}$ ) 


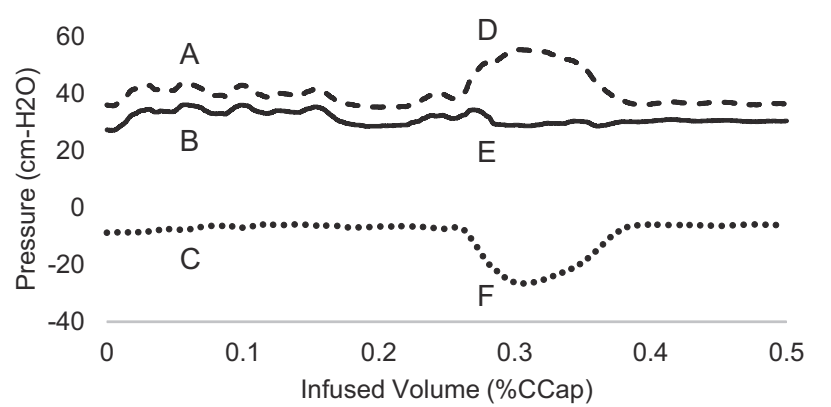

$\longrightarrow p_{\text {ves }} \quad---p_{\text {abd }} \quad \cdots \cdots \cdot p_{\text {det }}$

Fig. 2. Example tracings of measured $p_{v e s}$ and $p_{a b d}$ and calculated $p_{d e t}$ $\left(p_{\text {ves }}-p_{a b d}\right)$. The transient event that appears in both $p_{a b d}(\mathbf{A})$ and $p_{v e s}$ (B) does not appear in $p_{\text {det }}(\mathbf{C})$ and was not attributed to bladder activity. However, the event that increased $p_{a b d}(\mathbf{D})$ but not $p_{v e s}(\mathbf{E})$, significantly affected $p_{\text {det }}(\mathbf{F})$ and could have been incorrectly attributed to bladder activity if only $p_{\text {det }}$ had been considered.

which artificially alters the data, making it difficult or impossible to normalize. Furthermore, any statistically significant pattern in $p_{v e s}$ that correlates with repeat fills should be due to the repeat fill protocol and not transient abdominal events. For these reasons, the expected changes in wall tension due to strain softening were quantified using $p_{\text {ves }}$ instead of $p_{\text {det. }}$.

\section{UD Data Analysis}

Average $p_{\text {ves }}$ for each fill was normalized to average $p_{v e s}$ in Fil 1 for each patient for the filling ranges of 0-30\% CCap and $0-60 \%$ CCap. Mean normalized $p_{\text {ves }}$ and standard error were calculated for the cohort for each fill (Fig. 3). To estimate the change in the material properties of the bladder wall associated with reversible strain softening, we quantified dynamic elasticity as the ratio of the change in average $p_{v e s}$ between two fills to the change in percent capacity (Fig. 4). Statistical analyses of the data were performed via a two-way, paired Student's t-test. Significance was defined as $P<0.05$, and all values were reported as mean \pm standard error.

\section{RESULTS}

Five patients completed this pilot study and had results available for analysis $(n=5)$. All patients were female with average age $47 \pm 5$ years. Antimuscarinic use was documented in $2 / 5$ patients $(40 \%)$. There was no difference in bladder capacity when comparing maximum voided volume from 3-day void diary to CCap obtained in the clinical fill $(380 \pm 68 \mathrm{ml}$ vs. $563 \pm 75 \mathrm{ml}$, respectively, $P=0.12$ ). ICIq-OAB scores are shown in Table I.

In comparison to repeat Fill 1, Fills 2 and 3 displayed decreasing normalized $p_{\text {ves }}$ values from $0 \%$ to 30\% CCap, which reached statistical significance in Fill 3 (Fill 3: normalized $p_{\text {ves }}=0.46 \pm 0.11$ vs. Fill 1: 1.0, $P=0.008, n=5$ ) (Fig. 3A). This was attributed to strain softening during repeat passive filling and emptying. Active voiding occurred at the end of Fill 3, and $p_{\text {ves }}$ during Fill 4 returned to the baseline $p_{\text {ves }}$ produced during Fill 1 (Fill 4: normalized $p_{\text {ves }}=0.84 \pm 0.12$ vs. Fill $1: 1.0, p=0.29$ ), thereby demonstrating the reversibility of stain softening (Fig. 3A). Analysis of normalized average $p_{v e s}$ values from $0 \%$ to 60\% CCap for Fills 2-4 displayed consistent results (Fig. 3B). As a comparison, standard $\Delta \mathrm{V} / \Delta \mathrm{P}$ compliance over the $0-100 \%$ CCap range was $115 \pm 77 \mathrm{ml} / \mathrm{cmH}_{2} \mathrm{O}$ for Fill 0 and
$55 \pm 25 \mathrm{ml} / \mathrm{cmH}_{2} \mathrm{O}$ for Fill $3(P=0.56)$. This lack of significance for change in compliance (as compared to change in $p_{v e s}$ ) potentially highlights the improved diagnostic capabilities of our analytic technique.

Dynamic elasticity, the urodynamic correlate of adjustable preload tension, was calculated as amount of strain softening per percentage capacity ( $\Delta$ average $p_{\text {ves }}$ between passive fills/ $\Delta \%$ capacity). The loss of dynamic elasticity from Fills 1 to 3 (after strain softening) and the gain of dynamic elasticity from Fills 3 to 4 (after active voiding/strain softening reversal) are shown in Figure 4. The magnitude of the loss in dynamic elasticity attributed to strain softening and the magnitude of the gain in elasticity attributed to strain softening reversal were not statistically different $\left(0.28 \pm 0.09 \mathrm{~cm}-\mathrm{H}_{2} \mathrm{O} / \%\right.$ CCap vs. $-0.20 \pm 0.05 \mathrm{~cm}-\mathrm{H}_{2} \mathrm{O} / \%$ CCap, $P=0.49, \mathrm{n}=5$ ). These data indicate that the degree of strain softening caused during this protocol was reversed by voiding.

\section{DISCUSSION}

This pilot study identified the biomechanical bladder property of dynamic elasticity during a specially designed repeat fill-and-empty UD protocol in patients with OAB. Dynamic elasticity is the UD correlate of reversible strain softening (adjustable preload tension) previously identified in isolated human, rabbit, and mouse DSM tissue strips. ${ }^{4-12}$ Using our UD protocol, we were able to generate a novel metric for detrusor muscle function during the filling phase of micturition. We also demonstrated that standard $\Delta \mathrm{V} / \Delta \mathrm{P}$ compliance measurements do not effectively reflect the functionality of the detrusor muscle.

In the present protocol, progressive strain softening was observed in the $0-30 \%$ CCap and 0-60\% CCap ranges. This is because the bladder was strain softened by filling only to $30 \%$ in repeat Fill 1. Thus, any filling beyond $30 \%$ in subsequent fills would only be expected to provide additional strain softening to the volume range that had been previously reached. Therefore, our protocol was designed to demonstrate the biomechanical property of strain softening ( $p_{\text {ves }}$ Fill $1>2>3$ ) and reversibility after active voiding $\left(p_{\text {ves }}\right.$ Fill $4 \approx 1$ ) in the $0-30 \%$ CCap range as shown in Figure 3.

Tension sensors within the bladder wall are responsible for afferent nerve activity that leads to the sensation producing urgency. ${ }^{14}$ Therefore, we hypothesize that a derangement in the active processes regulating dynamic elasticity could contribute to the pathophysiology of an $O A B$ subtype. For example, a defect in dynamic elasticity that caused increased bladder wall tension during filling would be expected to result in increased afferent nerve activity. Thus, altered dynamic elasticity could be responsible for increased urgency at lower bladder volumes. Conversely, a defect in the dynamic elasticity mechanism associated with diminished strain softening reversal would result in a more floppy bladder and could reflect impaired contractility as seen in detrusor underactivity due to a decreased bladder preload observed in another study. ${ }^{15}$ Furthermore, straining to void, instead of a voiding contraction, would mimic our passive emptying protocol, resulting in a lack of strain softening reversal that could potentially exacerbate underactive bladder.

The current method for evaluating detrusor muscle stiffness during UDS is through the use of bladder compliance $(\Delta \mathrm{V} / \Delta \mathrm{P})$ calculations. ${ }^{1}$ The calculation, by definition, assumes a linear increase in pressure throughout bladder filling since only two time points (start of filling and cystometric capacity) are used. However, prior studies in both rats and humans have demonstrated the non-linear nature of compliance. ${ }^{16,17}$ Bladder 
A

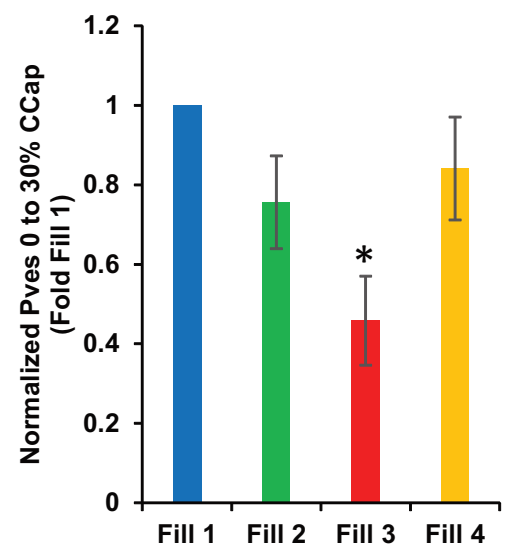

B

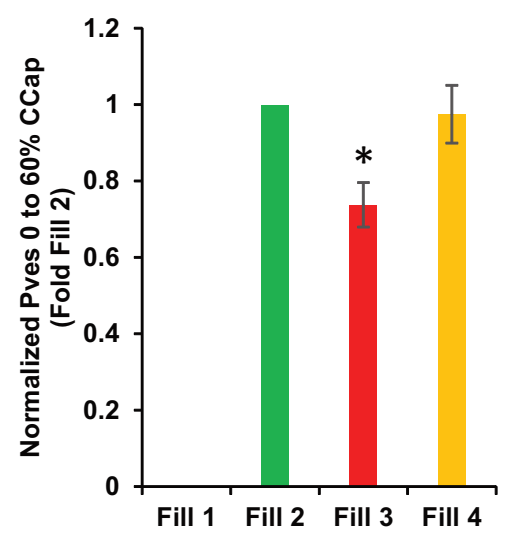

Fig. 3. Normalized average $\mathrm{p}_{\mathrm{ves}}$ for (A) 0-30\% CCap and (B) 0-60\% CCap for repeat fills $(\mathrm{n}=5)$. Decreasing $\mathrm{p}_{\mathrm{ves}}$ values were observed with subsequent Fills 2 and 3 (A) and Fill 3 (B). Fill $3 p_{\text {ves }}$ was significantly decreased compared to Fill $1 p_{\text {ves }}$ from $0 \%$ to $30 \%$ CCap (A) and compared to Fill 2 p ves from $0 \%$ to $60 \%$ CCap (B). Active voiding after Fill 3 caused $p_{\text {ves }}$ to return to baseline during Fill 4.

compliance has also been evaluated recently by the International Consultation on Incontinence (ICI). It was concluded that there is a wide range amongst healthy patients and that further standardization is required. ${ }^{18}$ These studies highlight the need for an improved metric, such as dynamic elasticity, which might better reflect underlying detrusor wall tension.

Examination of earlier studies involving repeated cystometry suggests the presence of dynamic elasticity in human bladders. In one of the earliest human cystometric studies, Simeone and Lampson showed a substantially more compliant pressurevolume relationship during cystometry following colon disimpaction and an additional increase in compliance $24 \mathrm{hr}$ later. ${ }^{19}$ Ockrim et al. demonstrated a statistically significant increase in all ICS sensory threshold volumes with repeat filling in a cohort of men with lower urinary tract symptoms (LUTS) undergoing three sequential repeat fills. ${ }^{20}$ Gupta et al. demonstrated significant increased volumes in both first sensation

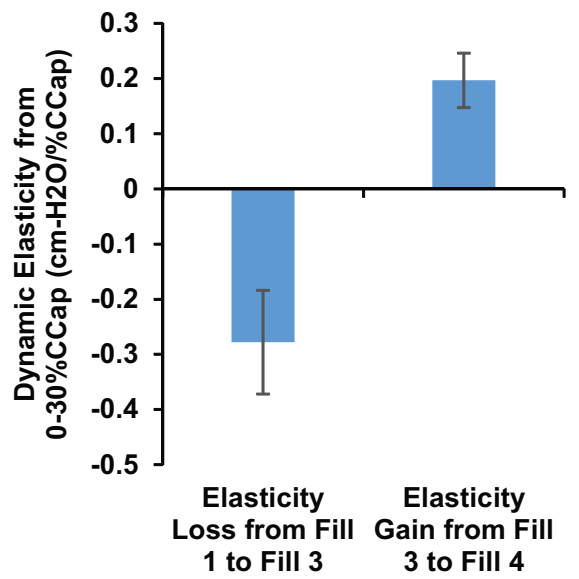

Fig. 4. Dynamic elasticity was defined as the ratio of the change in average $\mathrm{p}_{\mathrm{ves}}$ between two fills to the change in \% CCap. For the 0-30\% CCap range, the magnitude of the loss in elasticity from Fills 1 to 3 due to strain softening, $\left(p_{v e s} 3-p_{v e s} 1\right) / 30 \%$ Ccap, was not different from the magnitude of the gain in elasticity from Fills 3 to 4 due to strain softening reversal, ( $\left.p_{\text {ves }} 4-p_{\text {ves }} 3\right) / 30 \%$ CCap $(n=5)$. and first desire after sequential UD studies in healthy women with no signs of LUTS. ${ }^{21}$ These findings support our results that repeat fills strain soften the bladder and allow for increased volumes with repeat filling. Other groups have also previously shown that multiple UD parameters can vary with repeat filling. ${ }^{22,23}$

Smith et al. studied changes in compliance during the filling phase in both awake and centrally anesthetized mice which prevented the micturition reflex, requiring emptying of the bladder via pump. ${ }^{16}$ Thus, Smith's study examined compliance following both active voiding and passive emptying in mice, just as the present study does during human UD. Smith identified a reversible decrease in the absence of active detrusor contraction and attributed this finding to a decrease in micromotion activity thought to be suppressed when central control is intact. Prior studies in rabbit DSM have demonstrated that low amplitude rhythmic contractions (likely responsible for micromotion) are a mechanism for strain softening reversal (and thereby dynamic elasticity) in rabbit DSM. ${ }^{9}$ In pre-clinical studies, adjustable preload tension, the pre-clinical correlate of dynamic elasticity, has been attributed to cycling actomyosin crossbridges. ${ }^{4,6}$ Furthermore, adjustable preload tension has been shown to be under the influence of the rho-kinase pathway in multiple species, including humans, ${ }^{4,7,8,12}$ and may

TABLE I. Patient Characteristics

\begin{tabular}{lc}
\hline $\mathrm{n}$ & 5 \\
Age (years) & $47 \pm 5$ \\
Female & 5 \\
Male & 0 \\
Antimuscarinic use, n (\%) & $2(40)$ \\
ICIO-OAB score & \\
$3 a$ & $1.6 \pm 0.4$ \\
$3 b$ & $9.6 \pm 0.2$ \\
$4 a$ & $3.4 \pm 0.2$ \\
$4 b$ & $9.8 \pm 0.2$ \\
$5 a$ & $3.4 \pm 0.2$ \\
$5 b$ & $9.8 \pm 0.2$ \\
$6 a$ & $2.4 \pm 0.2$ \\
$6 b$ & $9.6 \pm 0.4$ \\
\hline
\end{tabular}


offer a target for future intervention in these types of voiding dysfunction.

Our calculation of dynamic elasticity using the change in pressure between fills is an approximation of the true change in elasticity, which is the change in the stress-strain relationship due to strain softening in the bladder wall. Other investigators have attempted to model bladder wall tension by combining viscoelastic detrusor strip stress-strain properties with UD pressure data. ${ }^{24,25}$ However, the benefit of the current investigation is that we were able to quantify dynamic elasticity simply by using our repeat filling and passive emptying protocol, without any increase in invasiveness for the patient. We recognize that these methods would be more robust if compared to attempts at directly or indirectly measuring wall tension or stress, and we have already begun to use 3D ultrasound to non-invasively measure bladder geometry which is necessary to calculate wall tension during urodynamic filling.

Limitations of this pilot study include small sample size, all female population, and all patients had OAB. Further investigation of volunteers without voiding dysfunction is needed to validate the findings of this study. Also, the potential confounding factors introduced during UD testing (non-physiologic filling rates and possible mucosal irritation from infusion catheter) could influence patient sensation thresholds for determining cystometric capacity.

\section{CONCLUSION}

This pilot investigation identified and measured the bladder biomechanical property of dynamic elasticity during UD in patients with $O A B$. This metric, determined using a specially designed repeat fill and empty protocol, represents the UD correlate to the previously identified property of adjustable preload tension (reversible strain softening) in preclinical investigations. The metric of dynamic elasticity offers substantial benefits beyond traditional $\Delta \mathrm{V} / \Delta \mathrm{P}$ compliance measurements. Furthermore, alterations in dynamic elasticity could be used in future studies to identify sub-sets of both $\mathrm{OAB}$ and underactive bladder that may be mediated by alterations in detrusor wall tension and in the identification of novel targets and drugs for the treatment of both conditions.

\section{REFERENCES}

1. Abrams P, Cardozo L, Fall M, et al. The standardisation of terminology of lower urinary tract function: Report from the standardisation sub-committee of the international continence society. Neurourol Urodyn 2002;21:167-78.
2. Landau EH, Jayanthi VR, Churchill BM, et al. Loss of elasticity in dysfunctional bladders: Urodynamic and histochemical correlation. J Urol 1994;152:702-5.

3. Metcalfe PD, Wang J, Jiao H, et al. Bladder outlet obstruction: Progression from inflammation to fibrosis. BJU Int 2010;106:1686-94.

4. Speich JE, Borgsmiller L, Call C, et al. ROK-induced cross-link formation stiffens passive muscle: Reversible strain-induced stress softening in rabbit detrusor. Am J Physiol Cell Physiol 2005;289:C12-21.

5. Southern JB, Frazier JR, Miner AS, et al. Elevated steady-state bladder preload activates myosin phosphorylation: Detrusor smooth muscle is a preload tension sensor. Am J Physiol Renal Physiol 2012;303:F1517-26.

6. Ratz PH, Speich JE. Evidence that actomyosin cross bridges contribute to "passive" tension in detrusor smooth muscle. Am J Physiol Renal Physiol 2010;298:F1424-35.

7. Colhoun AF, Speich JE, Dolat MT, et al. Acute length adaptation and adjustable preload in the human detrusor. Neurourol Urodyn 2015. doi: 10.1002/ nau. 22820

8. Speich JE, Southern JB, Henderson S, et al. Adjustable passive stiffness in mouse bladder: Regulated by Rho kinase and elevated following partial bladder outlet obstruction. Am J Physiol Renal Physiol 2012;302:F967-76.

9. Almasri $\mathrm{AM}$, Ratz $\mathrm{PH}$, Bhatia $\mathrm{H}$, et al. Rhythmic contraction generates adjustable passive stiffness in rabbit detrusor. J Appl Physiol (1985) 2010;108:544-53.

10. Speich JE, Dosier C, Borgsmiller L, et al. Adjustable passive length-tension curve in rabbit detrusor smooth muscle. J Appl Physiol (1985) 2007;102:1746-55.

11. Almasri AM, Ratz PH, Speich JE. Length adaptation of the passive-to-active tension ratio in rabbit detrusor. Ann Biomed Eng 2010;38:2594-605.

12. Shiomi H, Takahashi N, Kawashima $\mathrm{Y}$, et al. Involvement of stretch-induced Rho-kinase activation in the generation of bladder tone. Neurourol Urodyn 2013;32:1019-25.

13. Winters JC, Dmochowski RR, Goldman HB, et al. Urodynamic studies in adults: AUA/SUFU guideline. J Urol 2012;188:2464-72.

14. Kanai A, Andersson KE. Bladder afferent signaling: Recent findings. J Urol 2010;183:1288-95

15. Young JS, Johnston L, Soubrane $C$, et al. The passive and active contractile properties of the neurogenic, underactive bladder. BJU Int 2013;111:355-61.

16. Smith PP, Deangelis AM, Kuchel GA. Evidence of central modulation of bladder compliance during filling phase. Neurourol Urodyn 2012;31:30-5.

17. Coolsaet B. Bladder compliance and detrusor activity during the collection phase. Neurourol Urodyn 1985;4:263-73.

18. Wyndaele JJ, Gammie A, Bruschini $\mathrm{H}$, et al. Bladder compliance what does it represent: Can we measure it, and is it clinically relevant? Neurourol Urodyn 2011;30:714-22.

19. Simeone FA, Lampson RS. A cystometric study of the function of the urinary bladder. Ann Surg 1937;106:413-22.

20. Ockrim J, Laniado ME, Khoubehi B, et al. Variability of detrusor overactivity on repeated filling cystometry in men with urge symptoms: Comparison with spinal cord injury patients. BJU Int 2005;95:587-90.

21. Gupta A, Defreitas G, Lemack GE. The reproducibility of urodynamic findings in healthy female volunteers: Results of repeated studies in the same setting and after short-term follow-up. Neurourol Urodyn 2004;23:311-6.

22. Sorensen SS, Nielsen JB, Norgaard JP, et al. Changes in bladder volumes with repetition of water cystometry. Urol Res 1984;12:205-8.

23. Rosier PF, de la Rosette JJ, Koldewijn EL, et al. Variability of pressure-flow analysis parameters in repeated cystometry in patients with benign prostatic hyperplasia. J Urol 1995;153:1520-5.

24. Wagg A, Fry CH. Visco-elastic properties of isolated detrusor smooth muscle. Scand J Urol Nephrol Suppl 1999;201:12-8.

25. Korkmaz I, Rogg B. A simple fluid-mechanical model for the prediction of the stress-strain relation of the male urinary bladder. J Biomech 2007;40:663-8. 